\title{
Determinants for the use and de- implementation of low-value care in health care: a scoping review
}

\author{
Hanna Augustsson ${ }^{1,2^{*}} \mathbb{D}$, Sara Ingvarsson ${ }^{1}$, Per Nilsen ${ }^{3}$, Ulrica von Thiele Schwarz ${ }^{1,4}$, Irene Muli ${ }^{2}$, \\ Jessica Dervish ${ }^{2}$ and Henna Hasson 1,2
}

\begin{abstract}
Background: A considerable proportion of interventions provided to patients lack evidence of their effectiveness. This implies that patients may receive ineffective, unnecessary or even harmful care. However, despite some empirical studies in the field, there has been no synthesis of determinants impacting the use of low-value care (LVC) and the process of de-implementing LVC.
\end{abstract}

Aim: The aim was to identify determinants influencing the use of LVC, as well as determinants for deimplementation of LVC practices in health care.

Methods: A scoping review was performed based on the framework by Arksey and O'Malley. We searched four scientific databases, conducted snowball searches of relevant articles and hand searched the journal Implementation Science for peer-reviewed journal articles in English. Articles were included if they were empirical studies reporting on determinants for the use of LVC or de-implementation of LVC. The abstract review and the full-text review were conducted in duplicate and conflicting decisions were discussed until consensus was reached. Data were charted using a piloted data charting form and the determinants were inductively coded and categorised in an iterative process conducted by the project group.

Results: In total, 101 citations were included in the review. Of these, 92 reported on determinants for the use of LVC and nine on determinants for de-implementation. The studies were conducted in a range of health care settings and investigated a variety of LVC practices with LVC medication prescriptions, imaging and screening procedures being the most common. The identified determinants for the use of LVC as well as for deimplementation of LVC practices broadly concerned: patients, professionals, outer context, inner context, process and evidence and LVC practice. The results were discussed in relation to the Consolidated Framework for Implementation Research.

\footnotetext{
* Correspondence: hanna.augustsson@ki.se

'Procome Research Group, Medical Management Centre, Department of Learning, Informatics, Management and Ethics, Karolinska Institutet, SE 171 77 Stockholm, Sweden

¿Unit for Implementation and Evaluation, Center for Epidemiology and Community Medicine (CES), Stockholm Region, SE 17129 Stockholm, Sweden

Full list of author information is available at the end of the article
}

(c) The Author(s). 2021 Open Access This article is licensed under a Creative Commons Attribution 4.0 International License, which permits use, sharing, adaptation, distribution and reproduction in any medium or format, as long as you give appropriate credit to the original author(s) and the source, provide a link to the Creative Commons licence, and indicate if changes were made. The images or other third party material in this article are included in the article's Creative Commons licence, unless indicated otherwise in a credit line to the material. If material is not included in the article's Creative Commons licence and your intended use is not permitted by statutory regulation or exceeds the permitted use, you will need to obtain permission directly from the copyright holder. To view a copy of this licence, visit http://creativecommons.org/licenses/by/4.0/ The Creative Commons Public Domain Dedication waiver (http://creativecommons.org/publicdomain/zero/1.0/) applies to the data made available in this article, unless otherwise stated in a credit line to the data. 
(Continued from previous page)

Conclusion: The identified determinants largely overlap with existing implementation frameworks, although patient expectations and professionals' fear of malpractice appear to be more prominent determinants for the use and deimplementation of LVC. Thus, existing implementation determinant frameworks may require adaptation to be transferable to de-implementation. Strategies to reduce the use of LVC should specifically consider determinants for the use and de-implementation of LVC.

Registration: The review has not been registered.

Keywords: De-adoption, Disinvestment, De-implementation, Low-value care, Evidence-based

\section{Contributions to the literature}

- The study contributes the first synthesis of determinants, for the use of LVC as well as the de-implementation of LVC identified in the peer-reviewed literature.

- The study discusses similarities and differences between determinants for de-implementation and determinants for implementation in relation to the Consolidated Framework of Implementation Research (CFIR) and proposes that implementation determinant frameworks are useful also for deimplementation but may require some adaptations.

- Gaps in the knowledge concerning determinants for the use of LVC and de-implementation of LVC are identified and suggestions for further research are made.

\section{Background}

Use of health care practices (e.g. interventions, programmes and services) with little or no benefit to patients is a widespread problem [1]. Such practices are referred to as low-value care (LVC), which is "care that is unlikely to benefit the patient given the harms, cost, available alternatives, or preferences of the patient" [2]). Estimates show that $12-15 \%$ of patients receive at least one LVC practice a year [3] and $72 \%$ of US physicians stated that they normally prescribe unnecessary tests or procedures at least once a week [4].

The Choosing Wisely ${ }^{\oplus}$ campaign has produced over 550 recommendations for practices that are considered LVC, including use of antibiotics for upper respiratory infections, imaging for nonspecific low back pain and vitamin D testing [5]. Yet, practices identified as LVC in some clinical circumstances might be of high value in others. Thus, a key challenge is that interventions proven to be effective for specific patient populations are inappropriately applied to patients for whom benefit has never been demonstrated [6].

The use of LVC is costly. For example, the annual cost of LVC for the US Medicare population was estimated to be $\$ 8.5$ billion, which is almost $3 \%$ of total Medicare spending [7]. LVC is a concern both for individual patients and health care systems, and in order to provide evidence-based care to patients, there is a need to deimplement the use of LVC in addition to implementing evidence-based practices. De-implementation involves a structured and planned process using a set of activities to reduce or stop the use of LVC [8]. However, despite dissemination of numerous lists of LVC practices that should be abandoned, e.g. in the Choosing Wisely ${ }^{\circledR}$ campaign [9], the problem with LVC persists. This indicates that lists identifying LVC are not sufficient for these practices to be de-implemented [10].

There is a need to better understand the challenges of de-implementation, including what determinants influence the use of LVC and de-implementation of LVC practices. Determinants of implementation are well known from the current literature [11]. A number of determinants have been described in numerous frameworks, e.g. Consolidated Framework for Implementation Research (CFIR) [12], Promoting Action on Research Implementation in Health Services [13] and Theoretical Domains Framework [14]. These frameworks encompass determinants at multiple levels, from the individual professional, team and department to the organisational and societal levels. However, whether these frameworks are also applicable to categorise determinants of LVC use or de-implementing LVC practices is largely unknown since knowledge is lacking concerning the extent to which these determinants are the same as those pertaining to the implementation of evidence-based interventions $[8,15]$.

A recent review identified five studies that presented theories, models or frameworks specifically developed for de-implementation of LVC [16]. Four of these [17-20] encompassed determinants that might influence deimplementation of LVC. These determinants had been identified from an existing theory [17], from a literature review on social or behavioural constructs relevant to the de-implementation [20] and from empirical studies [18, 19]. None of these four studies was based on a literature review of determinants for de-implementation; thus, determinants for de-implementation of LVC has not been synthesised. Categorisations of determinants for the use of LVC and de-implementation of LVC could benefit the development of frameworks for de-implementation as well 
as adaptations of existing implementation frameworks to ascertain their applicability for de-implementation of LVC. Such a synthesis could also be important for exploring different strategy options to address the determinants to achieve de-implementation of LVC. Therefore, the aim of this review was to (1) identify and categorise determinants influencing the use of LVC and (2) identify and categorise determinants for de-implementation of LVC practices in health care. The identified determinants are discussed in relation to CFIR.

\section{Methods}

\section{Design}

We performed a scoping review methodology because it lends itself to address broader topics where studies using different designs and methods are synthesised [21]. The review process was based on the framework by Arksey and O'Malley [21], which outlines five stages: (1) identifying the research question, (2) identifying relevant studies, (3) study selection, (4) charting the data and (5) collating, summarising and reporting the results. The review is reported according to the PRISMA-ScR Checklist [22] (Additional file 1.).

\section{Protocol and registration}

The methodology has been described in a study protocol outlining the research project [23].

\section{Eligibility criteria}

We included empirical studies written in English and published in a peer-reviewed journal. Using the PCC mnemonic (Population, Concept, Context), recommended for scoping reviews [24], we specified that the content of the citations should describe determinants for the use of LVC practices and/or the de-implementation of LVC practices (concept) within health care (context). We did not specify a population since we were interested in determinants for the use and de-implementation of LVC in health care in general. All types of study designs were included, with the implication that studies reporting on supposed associations based on qualitative and descriptive data as well as associations based on quantitative data and statistical tests were included. To be included, a study needed to refer to a formal recommendation or a guideline stating that the practice was not recommended for a certain population/setting, i.e. of low value, to avoid judgements by the authors. We excluded studies that investigated determinants for potential overuse of a practice without identifying that the use was in fact inappropriate. All eligibility criteria are reported in Table 1.

\section{Information sources}

Searches were done in four electronic databases from different disciplines considered to be relevant to the
Table 1 Eligibility criteria for inclusion

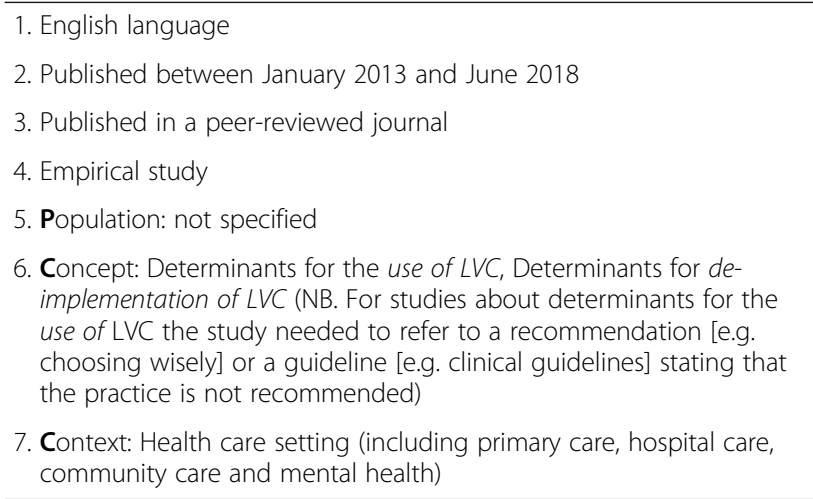

topic of LVC and de-implementation, such as biomedicine, health services research and nursing (MEDLINE, Embase, CINAHL and Web of Science). We also conducted hand searches of Implementation Science and of reference lists from relevant articles identified in the abstract screening.

\section{Search}

Potential keywords were identified through discussions among the authors, discussions with the Swedish Agency for Health Technology Assessment and Assessment of Social Services, from a review outlining terminology used for de-implementation [25] and through inspection of key papers focusing on de-implementation. The keywords were integrated into a search strategy in collaboration with librarians at the Karolinska Institutet library. The search terms were tested and refined three times to ensure that they captured 18 identified example papers and were discriminant enough to avoid yielding an overwhelming amount of citations. The librarians searched the databases from January 1, 2013, to June 4, 2018. This time limit was chosen because database searches showed an increasing number of papers from 2013 and onwards and due to a need to limit the number of studies since a vast amount of irrelevant studies was captured. The search strategy used in Web of Science is detailed in Table 2. Search strategies for all databases are included as an attachment (Additional file 2).

\section{Selection of sources of evidence}

All identified citations were imported into Rayyan, a web-based and mobile app that organises and facilitates the initial screening of titles and abstracts as well as the collaboration between reviewers [26]. The eligibility criteria were tested in multiple steps to ensure consensus among the reviewers. As a first step, four reviewers (HA, SI, IM, JD) tested the criteria on a sample of 40 abstracts. Inconsistencies were discussed and clarifications of the criteria were made. As a second step, reviewers piloted an additional 40 abstracts, which resulted in minor refinements of the criteria. Thereafter, five reviewers (HA, SI, 
Table 2 Search strategy used in Web of Science
Field labels
- TS/Topic = title, abstract, author keywords and Keywords Plus
- NEAR/ $x=$ within $x$ words, regardless of order
- * = truncation of word for alternate endings
\#1 TOPIC: (((abandon* OR contradict* OR deadopt* OR "de-adopt*" OR disadopt* OR "dis-adopt*" OR decommission* OR "de-commission*" OR deimplement* OR "de-implement*" OR delist* OR "de-list*" OR disinvest* OR "dis-invest" OR deprescript* OR deprescrib* OR divest* OR inapprop* OR ineffective* OR "low-value" OR obsole* OR outmoded OR overuse OR reallocate* $O R$ reassess ${ }^{*} O R$ "re-assess*" OR refute* $O R$ refuting $O R$ "re-invest*" OR "medical revers*" OR supersed* OR unlearn*) NEAR/3 (care OR clinic* OR device* OR drug OR drugs OR evidence* OR health OR healthcare OR medical OR medication* OR prescrib* OR procedur* OR technolog* OR therap* OR treat*)))

\#2 (((chang* or discontinu* or "dis-continu*" or decreas* or declin* or drop or reduc* or withdraw*) NEAR/1 ("use" or practice) NEAR/3 (care or clinic* $^{*}$ or device* or drug or drugs or evidence* or health or healthcare or medical or medication* or prescrib* or procedur* or technolog* or therap* or treat*)))

\#3 TS=("choosing wisely" or "priority setting") AND TS=(care or clinic* or device* $^{*}$ or drug or drugs or evidence* or health or healthcare or medical or medication* or prescrib* or procedur* or technolog* or therap* or treat*)

\#4 \#3 OR \#2 OR \#1

\#5 ((abandon* OR contradict* OR deadopt* OR "de-adopt*" OR disadopt* OR "dis-adopt*"

OR decommission* OR "de-commission*" OR deimplement* OR "deimplement*" OR delist* OR "de-list*" OR disinvest* OR "dis-invest" OR discontinu* OR "dis-continu*" OR deprescipt* OR deprescrib* OR divest* OR inapprop* OR ineffective* OR "low-value" OR obsole* OR outmoded OR overuse OR reallocate* OR reassess* OR "re-assess" OR refute* OR refuting OR "re-invest*" OR "medical revers*" OR supersed* OR unlearn* OR withdraw*) NEAR/3 (factor* OR barrier* OR engag* OR "evidencebased" OR facilitat* OR determinant* OR predict* OR model* OR framework* OR intervent* OR policy OR policies OR "practice pattern*" OR program* OR strateg* OR tool*))

\#6 \#5 AND \#4

Refined by: LANGUAGES: ( ENGLISH )

IM, JD, PN) applied the eligibility criteria to all citations. All abstracts were assessed independently by two reviewers. When all abstracts had been screened, the reviewers discussed the conflicting decisions. In cases where disagreement or uncertainty existed, the whole reviewer group discussed until a consensus was reached.

Thereafter, the five reviewers assessed the full texts of the included citations for final inclusion. This was done in pairs so that all articles were assessed independently by two reviewers. Conflicting decisions were discussed between the two reviewers, and in case of uncertainty, the article was discussed among the full reviewer group.

\section{Data charting}

A data charting form was developed. Five of the authors (HA, SI, IM, JD, PN) piloted the form by independently extracting data from three studies. The data were compared and inconsistencies were discussed, resulting in some changes of the form. A second test was made, in which the authors independently extracted data from two additional studies, which resulted in minor refinements of the form. The five authors then independently extracted data from the rest of the studies and continuously held meetings about any difficulties that emerged. Difficulties that were unable to be resolved were discussed by all authors. One of the authors (HA) read the extracted data from all 101 included studies to look for potential inconsistencies in what data had been extracted. This resulted in some minor additions of information for a few of the included studies.

\section{Data items}

Data charted related to general information about the citations (e.g. author) as well as to data related to the study aim (e.g. determinants for de-implementation) (Table 3). The first authors' affiliations were used as a proxy for country study origin when information about origin was missing.

\section{Synthesis of results}

An overview of the extent, nature and distribution of the included studies was reported in tables and text. Content analysis was conducted to inductively code and categorise the extracted information about the determinants of LVC and de-implementation of LVC. Inductive analyses is preferred when the knowledge base is limited [27]. Thus, the reason for applying an inductive approach instead of a deductive coding based on an existing implementation framework was that the knowledge about determinants for use of LVC and de-implementation of LVC is scarce and the extent to which determinants

Table 3 Items for data charting

Overview of items for data charting
a) Title
b) Journal
c) Authors
d) Year published
e) Country of study origin
f) Stated aim
g) Type of healthcare setting
h) Qualitative, quantitative, mixed methods
i) Study design
j) Method to assess the determinants (e.g. interviews, record review,
survey)
k) Study participants (type and number)
l) Type of LVC practice
m) Guideline or recommendation
n) De-implementation determinants
o) Use of LVC determinants


overlap with implementation determinants is unknown $[8,28]$. Using an inductive approach assured that determinants were identified, coded and categorised without the preconception that they were the same as for implementation. The coding entailed reading the extracted data and providing a code for the meaning of the determinants. For example, "cardiologists ordered inappropriate transthoracic echocardiography more frequently than other specialties" was assigned the code professional specialty. The coding of each determinant was conducted by at least two authors. Five of the authors (HA, SI, PN, UvTS, HH) then categorised the codes by scrutinising each individual code and sorting codes related to the same type of determinant into subcategories. For instance, the code professional specialty, defined as the health care professionals' medical specialisation, was sorted into the subcategory professional characteristics which address age, gender, years of experience in the profession, professional specialisation, medical school affiliation, lack of experience and personality. Thereafter, the subcategories were clustered into broader categories, e.g. the subcategory professional characteristics was sorted into a professional determinant category. All identified categories, subcategories and individual codes are outlined in Additional file 3.

Frequencies were used to summarise the number of studies reporting on specific determinants within the subcategories and categories. Information about the direction of the influence of the determinants, as reported in the included studies (both qualitative and quantitative studies), were indicated by referring to whether the determinant contributed to LVC use or was associated with lower use of LVC. The direction of the influence of determinants for de-implementation was indicated by specifying whether the determinant acted as a facilitator or barrier to de-implementation of LVC. The strengths of associations were not taken into account or reported since the scoping review encompassed studies with designs that do not allow for statistical tests of the associations, including qualitative studies. The synthesis and reporting of results were divided into two parts: (1) determinants for the use of $L V C$ practices and (2) determinants for de-implementation of $L V C$, i.e. a structured and planned process using a set of activities to reduce or stop the use of LVC.

\section{Results}

\section{Study selection}

The database search yielded 9542 citations (Fig. 1). An additional 186 citations were identified through searches in reference lists. After removing duplicates, 6570 citations remained for abstract screening. Of these, 586 citations were included for assessment in the full-text review. In total, 101 citations were included in the final review.

\section{Study characteristics}

Of the 101 studies, 92 focused on determinants for the use of $L V C$ and nine studies on determinants for de-implementation of $L V C$. These two types of studies are described separately below.

\section{Characteristics of studies on determinants for the use of LVC}

The majority of studies were conducted in the USA $(n=$ 54) and the most common settings were hospital and primary care (Table 4). Most studies had a quantitative design and collected data through record reviews and surveys. Data were collected from professionals in 28 studies, patients in 51 studies and from professionals as well as patients in 13 studies.

The included studies investigated a range of LVC practices (Table 5). The majority of studies $(n=73)$ focused on one LVC practice. In total, 165 types of LVC practices were investigated.

The practices were sorted into eight categories. The most common LVC was medication prescriptions $(n=69)$, encompassing non-indicated prescription of antibiotics, potentially inappropriate medications and antipsychotics. A total of 41 practices concerned imaging (e.g. imaging for back pain) and 20 practices were related to screening, including vitamin $\mathrm{D}$ deficiency screening for low-risk patients and ECG screening in general medical examinations. Fourteen practices concerned cardiovascular testing and imaging and varying diagnostic tests (e.g. thyroid function test in asymptomatic patients and unnecessary pre-operative tests) were the focus of 11 studies. The remaining practices concerned non-surgical and surgical procedures $(n=3)$ and radiation therapy $(n=2)$. Five studies could not be categorised in any of these categories and were labelled other.

\section{Characteristics of studies on determinants for de- implementation of LVC}

Of the nine studies investigating determinants for deimplementation of LVC, five were conducted in the USA (Table 6). Most studies were conducted in primary care and hospital settings. The majority of studies $(n=5)$ had a quantitative design and collected data from professionals $(n=7)$.

Most de-implementation studies focused on single LVC practices $(n=4)$ (Table 7). Two of the studies concerned multiple LVC practices and two investigated LVC practices in general. A total of $21 \mathrm{LVC}$ practices were identified and the most studied type of LVC practice was medication prescriptions $(n=7)$, which included practices such as non-indicated prescription of antibiotics.

\section{Determinants for the use of LVC}

The identified determinants were related to patients, professionals, outer context, inner context, process and 


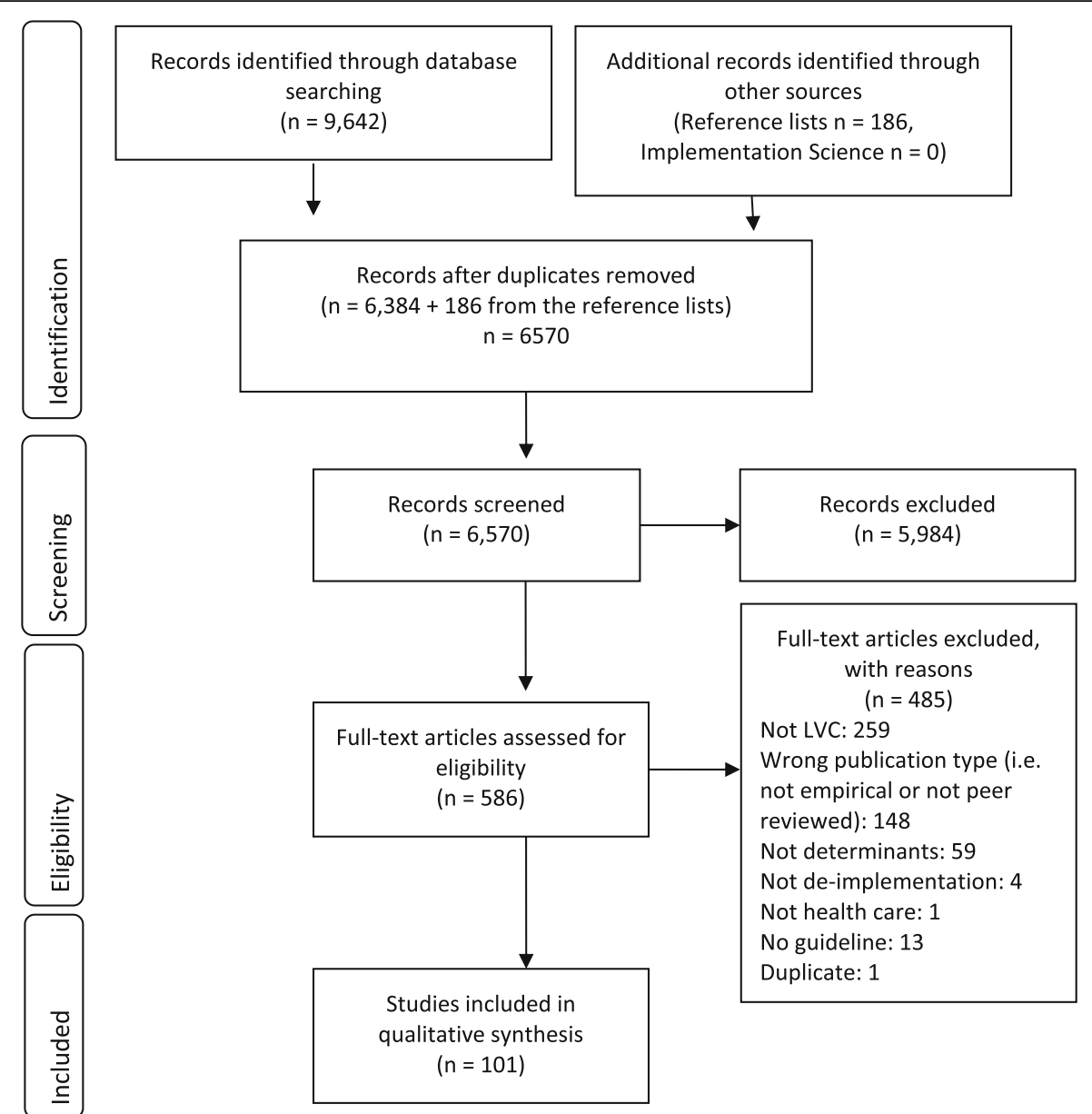

Fig. 1 PRISMA flow diagram

evidence and LVC practice. These categories include a number of subcategories (Table 8). A table with all identified determinants in each study is provided in Additional file 4.

\section{Patient determinants}

Patient-related determinants were the most commonly identified type of determinant. The studies reported a total of 72 determinants that contributed to the use of LVC and 12 determinants that contributed to lower use of LVC. The category consists of four subcategories.

Patient characteristics were identified as a determinant contributing to the use of LVC in 37 studies and to lower use in 10 studies and included, e.g. age, gender, ethnicity and socio-economic factors. There was no consistent pattern as to the positive or negative influence on LVC. For instance, older age was most often associated with use of LVC (e.g. [29-31]), but there were also studies where younger age was associated with LVC (e.g. $[32,33])$. Patient health condition was found to contribute to use of LVC in 17 studies and to lower use in two studies. Patient health condition included determinants such as severity of illness (e.g. [34]) and characteristics of the disease (e.g. [35]). Patient expectations, e.g. patients who requested non-indicated prescriptions, increased the occurrence of LVC in 16 studies (e.g. [36, 37]). Also expectations from relatives $(n=2)$ contributed to the use of LVC $[38,39]$.

\section{Professional determinants}

In total, 47 professional determinants contributed to use of LVC and 12 determinants to lower use of LVC. The category included three subcategories.

Professional characteristics encompassed determinants such as age, gender, medical speciality, professional training and personality, which were identified in 24 studies to contribute to the use of LVC and found to be related to lower use of LVC in seven studies. As with patient characteristics, the results were inconsistent regarding age, gender and length of experience of the professionals. Lack of or inadequate training was consistently linked to use of LVC (e.g. [40, 41]). 
Table 4 Study characteristics for the studies investigating determinants for the use of LVC

\begin{tabular}{|c|c|c|}
\hline Study aspect & Number of studies (total $n=92$ ) & $\%$ of total number of studies \\
\hline \multicolumn{3}{|l|}{ Healthcare setting } \\
\hline Hospital & 34 & 36.9 \\
\hline Primary care & 24 & 26.1 \\
\hline Multiple settings & 17 & 18.5 \\
\hline Aged care & 7 & 7.6 \\
\hline Non-specific setting & 8 & 8.7 \\
\hline Other $^{a}$ & 2 & 2.2 \\
\hline \multicolumn{3}{|l|}{ Method } \\
\hline Quantitative & 78 & 84.8 \\
\hline Qualitative & 11 & 12 \\
\hline Mixed methods & 3 & 3.2 \\
\hline \multicolumn{3}{|l|}{ Study design } \\
\hline Retrospective review & 40 & 43.5 \\
\hline Cross-sectional & 32 & 34.8 \\
\hline Cohort & 4 & 4.3 \\
\hline Experimental & 2 & 2.2 \\
\hline Prospective observations & 3 & 3.2 \\
\hline Other ${ }^{c}$ & 11 & 12 \\
\hline \multicolumn{3}{|l|}{ Data collection method ${ }^{b}$} \\
\hline Record review & 50 & 54.3 \\
\hline Survey & 30 & 32.6 \\
\hline Interviews & 11 & 12 \\
\hline Focus groups interviews & 3 & 3.3 \\
\hline Administrative data & 2 & 2.2 \\
\hline \multicolumn{3}{|l|}{ Participants in the studies } \\
\hline Healthcare professionals & 28 & 30.5 \\
\hline Patients & 51 & 55.4 \\
\hline Healthcare professionals and patients & 13 & 14.1 \\
\hline
\end{tabular}

anentistry, Informal healthcare sector

${ }^{\mathrm{b}}$ Total percentage exceeds $100 \%$ as some studies used more than one data collection method.

${ }^{c}$ Qualitative interview studies

Table 5 Type of LVC practices investigated in the studies examining determinants for the use of LVC

\begin{tabular}{lll}
\hline Type of LVC practice & Number of practices (total $\boldsymbol{n}=\mathbf{1 6 5})^{\mathbf{a}}$ & \% of total number of practices \\
\hline Medication prescriptions & 69 & 41.8 \\
Imaging & 41 & 24.9 \\
Screening & 20 & 12.1 \\
Cardiovascular testing and imaging & 14 & 8.5 \\
Diagnostic tests & 11 & 6.7 \\
Procedure (non-surgical and surgical) & 3 & 1.8 \\
Radiation therapy & 2 & 1.2
\end{tabular}

${ }^{a}$ The number of studies exceeds the total number of studies included in the review and the percentage exceeds $100 \%$ because some studies investigated several LVC practices

${ }^{b}$ E.g. feeding tubes in dementia patients, non-indicated foley catheter use 
Table 6 Study characteristics for studies investigating determinants for de-implementation of LVC

\begin{tabular}{|c|c|c|}
\hline Study aspect & Number of studies (total $n=9$ ) & $\%$ of total number of studies \\
\hline \multicolumn{3}{|l|}{ Healthcare setting } \\
\hline Hospital & 3 & 33.3 \\
\hline Primary care & 3 & 33.3 \\
\hline Policy & 2 & 22.2 \\
\hline Anticoagulation clinic & 1 & 11.1 \\
\hline \multicolumn{3}{|l|}{ Method } \\
\hline Quantitative & 5 & 55.6 \\
\hline Mixed method & 3 & 33.3 \\
\hline Qualitative & 1 & 11.1 \\
\hline \multicolumn{3}{|l|}{ Study design } \\
\hline Cross-sectional & 6 & 66.7 \\
\hline Experimental & 1 & 11.1 \\
\hline Longitudinal & 1 & 11.1 \\
\hline Three-round Delphi & 1 & 11.1 \\
\hline \multicolumn{3}{|l|}{ Data collection method ${ }^{a}$} \\
\hline Survey & 7 & 77.8 \\
\hline Interviews & 3 & 33.3 \\
\hline Record review & 1 & 11.1 \\
\hline Delphi & 1 & 11.1 \\
\hline \multicolumn{3}{|l|}{ Participants in the studies } \\
\hline Healthcare professional & 5 & 55.6 \\
\hline Healthcare professionals and other stakeholders & 2 & 22.2 \\
\hline Patients & 2 & 22.2 \\
\hline
\end{tabular}

${ }^{a}$ Total percentage exceeds $100 \%$, as some studies used more than one data collection method

Professionals' knowledge of $L V C$ both contributed to the use of LVC $(n=6)$ and protected against LVC use $(n=$ $3)$. Being aware of a guideline advising against the use of the practice was related to less LVC (e.g. [42]), whereas not having access to guidelines (e.g. [43]) or misinterpreting guidelines [44] increased LVC. A lack of knowledge about cost-effectiveness [45] and lack of cost-awareness [46] were linked to the use of LVC.

The majority of determinants in professionals' expectations, attitudes and behaviours were related to higher use of LVC $(n=17)$. Determinants included professionals' fear of malpractice, which was a commonly identified reason for providing LVC. For instance, the risk of missing a diagnosis was perceived to be more important than the risk of exposing patients to unnecessary radiation in relation to LVC imaging [47]. Another related determinant was fear of liability or litigation as a reason for using LVC, e.g. unnecessary diagnostic tests [48]. Professionals' expectations, attitudes and behaviours also included professionals' desire to meet patient requests (e.g. [45, 49]), attitudes toward or beliefs about existing evidence and guidelines (e.g. [50]) and the habit of using a practice (e.g. [46, 51]). Two studies identified determinants that were linked to lower use of LVC: professionals' cost-consciousness [42] and perceived usefulness of guidelines as well as self-efficacy with regard to discussing LVC and costs [52].

Table 7 Type of LVC practices investigated in the studies examining determinants for de-implementation of LVC

\begin{tabular}{lll}
\hline Type of LVC practice & Number of practices (total $\boldsymbol{n}=\mathbf{2 1}$ ) & \% of total number of practices \\
\hline Medication prescriptions & 7 & 33.3 \\
Screening & 5 & 23.8 \\
Imaging & 4 & 19 \\
Tests & 3 & 14.3 \\
Procedures & 2 & 9.5 \\
\hline
\end{tabular}


Table 8 Identified determinants for the use of LVC and de-implementation of LVC

Studies on determinants for LVC use total $n=92$ Determinants

\section{Total no of No (\%) of} studies including the the determinant determinant to determinant to LVC use
Studies on determinants for de-implementation total $n=9$

Total no No (\%) of studies No (\%) of studies
of studies with the determinant with the determinant including the as a facilitator of de- as a barrier to dedeterminant implementation implementation

\section{Patient determinants}

Patient characteristics $\quad 39$

37 (94.9)

$10(25.6)$

Patient health condition 19

17 (89.5)

$2(10.5)$

Patient expectations

16 (100)

Patient knowledge

Expectations from

relatives

\section{Professional determinants}

characteristics

Professional knowledge 9

of LVC

Professionals

and behaviours

Professionals' memory

\section{Outer context determinants}

Location
Economy
Outer context
characteristics
Patient volume
Policy and political
support
Marketing
Time
Pressure from suppliers

\section{Inner context determinants}

Setting characteristics
Work/care process
Staff composition
Organisational structures
related to the LVC
Interaction between
professionals
Culture
Patient-provider
communication/
interaction

\section{Process determinants}

Strategies
De-implementation
process

Evidence and LVC practice determinants 
Table 8 Identified determinants for the use of LVC and de-implementation of LVC (Continued)

\begin{tabular}{|c|c|c|c|c|c|c|}
\hline \multicolumn{4}{|c|}{ Studies on determinants for LVC use total $n=92$} & \multicolumn{3}{|c|}{ Studies on determinants for de-implementation total $n=9$} \\
\hline Determinants & $\begin{array}{l}\text { Total no of } \\
\text { studies } \\
\text { including the } \\
\text { determinant }\end{array}$ & $\begin{array}{l}\text { No (\%) of } \\
\text { studies linking } \\
\text { the determinant } \\
\text { to LVC use }\end{array}$ & $\begin{array}{l}\text { No }(\%) \text { of studies } \\
\text { linking the } \\
\text { determinant to } \\
\text { low LVC use }\end{array}$ & $\begin{array}{l}\text { Total no } \\
\text { of studies } \\
\text { including the } \\
\text { determinant }\end{array}$ & $\begin{array}{l}\text { No }(\%) \text { of studies } \\
\text { with the determinant } \\
\text { as a facilitator of de- } \\
\text { implementation }\end{array}$ & $\begin{array}{l}\text { No (\%) of studies } \\
\text { with the determinant } \\
\text { as a barrier to de- } \\
\text { implementation }\end{array}$ \\
\hline Characteristics of the LVC & 2 & $2(100)$ & & - & & \\
\hline $\begin{array}{l}\text { Negative consequences } \\
\text { of reducing LVC for the } \\
\text { professional }\end{array}$ & - & & & 1 & & $1(100)$ \\
\hline $\begin{array}{l}\text { Characteristics of } \\
\text { alternative practice }\end{array}$ & 2 & $2(100)$ & & 1 & & $1(100)$ \\
\hline Other $^{a}$ & 3 & $3(100)$ & & - & & \\
\hline
\end{tabular}

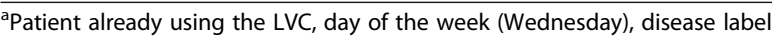

\section{Outer context determinants}

The outer context included determinants related to the social, political and geographical context of health care, comprising six sub-categories. In total, 42 determinants related to a higher use of LVC and 15 determinants related to lower use were reported.

Location referred to the type of geographical area, e.g. metropolitan, urban, suburban and rural as well as different identified regions. There was no clear pattern concerning what type of geographical area was linked to higher or lower use of LVC, i.e. this differed in the included studies (e.g. [33, 50, 53, 54]). Economy included determinants contributing to LVC use $(n=14)$ as well as determinants associated with lower use of LVC ( $n=$ 4) and was related to the financing of health care and financial incentives. In the majority of the studies, having private health insurance was linked to receiving LVC, when compared to other insurance types [53-56]. However, having Medicaid or being uninsured was also associated with receiving LVC [57]. Although inappropriate prescribing measures were more common in uninsured patients and patients with Medicaid, compared to privately insured patients, computed tomography/magnetic resonance imaging for back/neck pain was used to a higher extent for patients with private insurance [58]. The category also included studies on reimbursement models and financial incentives. Incentives for using LVC [48], fee-for-service funding [59] and patients not sufficiently sharing in the cost of their health care [45] were related to a higher use of $\mathrm{LVC}$, and a decreased reimbursement for a LVC practice was associated with lower use of the practice [60].

Outer context characteristics included determinants related to the patient or professional population in the specific area for the study. Such characteristics were related to higher as well as lower use of LVC practices in four studies, respectively. For instance, municipalities with lower socioeconomic status [61] and regions with high health care consumption [62] were found to have a higher extent of LVC. A higher specialist to primary care ratio [62] and specialist density [63] were associated with higher use of LVC services, and higher physician group concentration (a measure of market competition) was linked to lower use of LVC practices [62]. Patient volume influenced LVC use in four studies. For example, lower patient volume at practice level was related to inappropriate prostate cancer imaging [64] and inappropriate breast cancer imaging as assessed by clinical vignettes [50]. However, when it came to individual physicians' patient volume, a higher volume of patients per day was related to a higher use of LVC [65].

Policy and political support were found to influence the use of LVC in three studies. Having a policy concerning restricted LVC use was linked to lower use $[59,66]$, and a lack of political support, on the other hand, was perceived to be a barrier to reduce overuse [67]. Marketing in the form of direct-to-consumer advertising about drugs and treatments [45], promotion of screening directed to the population [59] and interests of health care companies or the pharmaceutical industry [48] were identified as influencing the use of LVC in three studies. Time was addressed in four studies that investigated the fluctuation of LVC use over time, i.e. from 1 year to another. These studies showed that the use of specific LVC practices was inconsistent and increased or decreased from one year to another without any clear trend over time [33, 64, 68, 69].

\section{Inner context determinants}

The inner context comprised determinants related to the structural and social environments of the health care settings, e.g. hospitals, clinics and care centres, and entailed seven subcategories. Fifty determinants were linked to a higher use of LVC and 10 related to lower use.

Setting characteristics included studies that compared the extent of LVC between different types of settings, e. g. hospital-based practices compared with communitybased practices (e.g. [70-72]), usually without providing information about potential reasons for the varying use. 
Work/care process included lack of care continuity (e.g. number of handoffs and having several care providers) which was linked to a higher use of LVC practices (e.g. [73-76]). Perceived lack of time and time pressure when performing work tasks were associated with higher use of LVC (e.g. [36, 77]). Other identified determinants included perceived inaccessibility of decision support [44], individual rather than clinic-based decision-making [59] and lack of feedback/accountability [49], which all contributed to more LVC. Multidisciplinary rounds [75] and teamwork [40] were found to be related to lower use of LVC. The subcategory Staff composition included inadequate staffing levels [40] and solo practice $[60,78]$ which were linked to higher use of LVC.

Organisational structures related to the $L V C$ referred to structures in the organisation that provided incentives for or facilitated using LVC, such as the ownership of equipment that were used for providing the LVC practice $[51,79]$ and structures that made it easy to order repeating/standing lab tests [46]. Interaction between professionals mainly included the recommendation, expectation or request by another professional or specialist to provide the LVC practice $[37,59,80]$. Also, a lack of communication between professionals was identified as a determinant for the use of LVC in one study [44]. Different types of culture were identified to contribute to the use of LVC in three studies. A screening culture was considered to influence prostate-specific antigen (PSA) testing in Australia [59], a hierarchical culture with a lack of debate was perceived to contribute to LVC in primary care [48] and a culture of poor cost consciousness was perceived to contribute to unnecessary inpatient laboratory tests [46]. Patient-provider communication/interaction was identified as influencing the use of LVC in two studies, describing challenges that care providers faced when communicating with patients about unnecessary antibiotics [43] and a paternalistic doctor-patient relationship that contributed to an environment in which potentially inappropriate prescribing can occur [81].

\section{Process determinants}

The process involved determinants related to processes for managing LVC and was the least common category reported $(n=4)$. This category included the subcategory strategies, which referred to strategies used to limit the use of LVC. The identified strategies were linked to lower use of LVC and included having processes and routines for managing LVC issues (meetings, review and communication with care recipients and relatives) [82]; managerial priorities for non-pharmacological management [40] patient education, provider education and decision support [43]; and medication reviews [41].

\section{Evidence and LVC practice determinants}

Determinants related to the evidence and the LVC practice were linked to LVC use in 11 studies and included three sub-categories.

Evidence included conflicting guidelines [50,59] and inappropriate or inapplicable guidelines for the patient group [52, 77], which led to more LVC. Furthermore, professionals' belief that the LVC was, in fact, effective, despite guidelines recommending against its use was reported as a reason for using LVC (e.g. [41]). Characteristics of the $L V C$ was a determinant when the LVC practice had advantages, such as being easy to distribute [41] or lacking perceived negative consequences [41, 47]. Characteristics of alternative practice (i.e. an alternative to the LVC practice), such as when the alternative lacked guidelines [41] or access [83], was also a determinant for LVC.

\section{Determinants for de-implementation of LVC}

The nine studies concerning determinants for deimplementation of LVC were related to the same broad categories as the determinants for using LVC. Each category consisted of a number of subcategories (Table 8). A table with all identified determinants in each study is provided in Additional file 4.

\section{Patient determinants}

Patient determinants typically acted as barriers to deimplementation. The most reported patient determinant was expectations from patients, which was as a barrier to de-implementation in five studies [84-88]. This included patients' opposition to de-implementation [84], patients' request for tests and treatments $[85,86]$ and challenges overcoming patients' preferences and values and community standards of care [88]. Patient knowledge was both a facilitator and a barrier to de-implementation. Patients' being aware of the negative consequences of LVC facilitated de-implementation [89], whereas a lack of knowledge acted as a barrier [88].

\section{Professional determinants}

Professional determinants was the most common category. All studies described barriers to de-implementation. The most common subcategory was professionals' expectations, attitudes and behaviour which consisted of clinicians resistance to change [84], fear of malpractice due to a "malpractice system" [85], lack of interest in saving money [90] and fear of litigation [87, 88]. Professionals' knowledge included one study that described a gap in professionals knowledge about their actual use of the LVC practice which meant that they overestimated to what extent they had reduced their use [86]. The subcategory professionals' memory, had to do with clinicians' forgetting to assess patients' eligibility for a decreased use of a practice [86]. 


\section{Outer context determinants}

Outer context determinants were found to both facilitate and hinder de-implementation and comprised three subcategories.

Concerning policy and political support, having a clear rationale for change [91] facilitated de-implementations, and a weak political willingness [84] hindered deimplementation. One study found that a lack of physicians' influence on policy concerning LVC practice had a positive influence on the intention to stop using the practice [90]. The same study found that pressure from suppliers to use the LVC practice had a positive relation to de-implementation. According to the authors, one possible explanation is that physicians may only perceive these to be barriers when they have the intention to stop using the LVC practice and feel hindered by these factors [90]. The subcategory economy encompassed one study showing that payment policies and performance measures that rewarded more services acted as barriers to de-implementation [85].

\section{Inner context determinants}

Inner context determinants were found to hinder as well as facilitate de-implementation and were related to four subcategories.

In the subcategory setting characteristics, one study found that academic medical centres performed better in relation to de-implementation, compared to for-profit and not-for-profit centres [92]. This was connected to the organisational culture, i.e. norms, values and incentives, of academic medical centres, which seemed to influence the behaviour not only of their regular employees, but also the freelancers working at the centres [92]. Work/care process was a potential barrier to deimplementation $(n=3)$ and included time pressure in general $[86,88]$, lack of time for shared decision-making [85] and lack of tools for shared decision-making [88]. Interaction between professionals $(n=1)$, i.e. the number of tests and treatments recommended by specialists, was a barrier to de-implementation [85].

\section{Process determinants}

Two studies identified process determinants related to the subcategory de-implementation process. A Delphi study proposed several determinants that may both hinder and facilitate de-implementation [91], with the most important being strength of executive leadership, strength of clinical leadership, quality of communication, clarity of specific aims and objectives, extent of cultural and behavioural change, attention to human aspects of process of change, quality of project management, availability of resources to support decision-making and implementation processes, quality of strategic planning and training of staff [91]. The other study identified complexity of the de-implementation process as a barrier when a specialised process or extensive patient education was required to reduce the LVC practice [86].

\section{Evidence and LVC practice determinants}

One study identified a lack of alternative practice to replace the LVC practice as a barrier to deimplementation [90]. Another study identified a lack of reliable and easily available information concerning current clinical practice and on safety, effectiveness and costs of health care interventions as a barrier for deimplementation [84].

\section{Discussion}

This scoping review included a total of 101 studies of which the majority reported on determinants for the use of LVC $(n=92)$ and a limited number on determinants for de-implementation $(n=9)$. Our analysis resulted in six categories: patient determinants, professional determinants, inner context determinants, outer context determinants, process determinants and evidence and LVC practice determinants.

We used an inductive approach to develop the determinant categories although there are numerous frameworks to choose from within implementation science. However, while findings from implementation of evidence-based interventions might inform de-implementation research, it cannot be granted that knowledge is easily transferable between the two areas $[8,28]$. Indeed, Davidson et al. [93] have called for a distinct de-implementation science that would "recognize and identify problem areas of low-value and wasteful practice, carry out rigorous scientific examinations of the factors that initiate and maintain such practices and then employ evidence-based interventions to extinguish these practices".

The broad categories of determinants for the use and de-implementation of LVC largely overlap with implementation determinants as described in CFIR [12]. CFIR provides a relevant comparison because it is one of the most comprehensive and most widely applied frameworks [94]. CFIR is broadly similar to many other implementation determinant frameworks as there is considerable consensus as to what the main types of implementation determinants are [11]. CFIR encompasses five determinant domains (our categories in brackets): intervention characteristics (evidence and LVC practice determinants). inner setting (inner context determinants), outer setting (outer context determinants), characteristics of individuals (professional determinants) and process (process determinants [12]. In our review, patient determinants make up a unique category whereas in CFIR, patient needs and resources is a sub-domain that is part of the outer setting domain. The representation of patient determinants as a category of its own provided the most accurate representation of the data 
since patient determinants was a prominent determinant in the included studies. Furthermore, we identified two determinants that were more prominent with regard to the use of LVC and de-implementation of LVC than for implementation: patient expectations and professionals' fear of malpractice.

Patient expectations are less commonly addressed in implementation frameworks (e.g. [12, 95, 96]). One possible reason for this difference may be that deimplementation involves reducing or stopping known practices that the patients are aware of and may previously have received. Implementation, on the other hand, often deals with implementing new practices that patients may not be aware of and, consequently, do not request. The influence of patients on the use of LVC should also be understood in light of factors influencing patients, e.g. advertisements and tests or treatments they are used to receiving. These factors can function as drivers as well as inhibitors of the use of LVC. For instance, extensive measures are undertaken to reduce antimicrobial resistance, including campaigns to raise awareness in the population [97]. By contrast, media attention to the positive effects of vitamin $\mathrm{D}$ has been suggested to have contributed to increased vitamin D lab tests [98], despite guidelines recommending against screening in the general population [99].

Professionals' fear of malpractice, for instance, due to missing a diagnosis, or of becoming the target of litigation, was another determinant found in this review that is rarely identified as an implementation determinant. It may seem paradoxical that fear of malpractice was a driver of using LVC since providing LVC may be directly harmful to patients. However, a potential explanation is that some LVC practices, e.g. computed tomography scans of the head in patients with minor head injury [47], were considered relatively harmless, relative to the risk of missing a serious diagnosis. Fear of litigation is likely a more prominent determinant in countries that have a system in which such legal actions are fairly common.

Professionals' attitudes and behaviours have been identified to influence both implementation [12] and deimplementation, but the reasons and mechanisms for how these influences operate may differ between the two processes, as has also been proposed by Norton and Chambers [100]. Reducing LVC may be difficult when health care professionals have developed familiarity and expertise with certain practices, to the extent that such practices become intertwined with their identity, position and status [101-103]. Helfrich et al. [104] suggest that attempts at de-implementation may be perceived by health care professionals as an attack or infringement on their personal prerogative, which can actually increase their commitment to the current LVC practice. There are also organisational and system-level influences that may inhibit efforts to reduce the use of LVC. The culture of teams, professions and organisations constitutes a stabilising influence by imposing norms, values, beliefs and assumptions that become internalised and tacitly held among members of the groups, thus functioning as "collective habits" shared among groups of people [105].

The process category was the least reported category in this review with only two broad subcategories (strategies and de-implementation process) of determinants identified. CFIR, on the other hand, outlines several different and more specific determinants (e.g. planning, engaging, executing, reflecting and evaluating). This makes it challenging to draw any firm conclusions concerning the extent to which process determinants for the use and de-implementation of LVC overlap with process determinants for implementation. In general, there is a paucity of research on de-implementation processes which may be due to difficulties of defining, measuring and evaluating this domain [106].

Consistent with CFIR, this review shows that determinants for use of LVC and de-implementation of LVC exist on all levels of the system, from the individual patient and professional to the work group, organisation and the wider health care system. Individual-level determinants were the most commonly identified determinants. A majority of the included studies were retrospective record reviews, which reported on associations between different determinants and the use of LVC. These studies were not designed to explore a range of potential determinants; rather, the focus was on the limited number of variables available in medical records, such as patient characteristics. This means that important determinants may have been neglected.

Despite the fairly large focus on individual-level determinants, the included studies show that individual professionals' decision to provide or not provide LVC is affected by their context and the interactions with patients and with other professionals. This suggests that it is important to provide a work environment (e.g. peersupport) and work process (e.g. decision support) in which individual professionals are supported and have the prerequisites to resist LVC.

This underscores the importance of viewing health care systems in holistic terms, because the use of LVC likely depends on combinations of determinants. Furthermore, the multi-level determinants impacting the use of LVC and de-implementation of LVC point to the relevance of accounting for determinants at different levels when planning strategies to reduce the use of LVC. It is important to avoid an overly reductionist approach, studying the impact of different determinants in isolation of each other, since this neglects the fact that two or more seemingly unimportant determinants may 
create powerful effects if they are combined and potentially strong influences may combine to generate weak effects [5].

\section{Knowledge gaps and implications}

The findings imply that existing implementation determinants are useful also for assessing determinants for the use and de-implementation of LVC. However, the results also show that adaptations may be required. We propose that greater consideration should be placed on how patient expectations and professionals' fear of malpractice influence the use of LVC. These determinants should also be considered when planning strategies to de-implement LVC. The findings also imply that health care organisations must consider determinants at different levels when attempting to reduce LVC and that strategies solely targeting individual professionals may not be sufficient for reducing LVC.

Several areas in need of further research can be identified. First, the results point to a dearth of studies on determinants for the process of de-implementing LVC. Second, there is a need for studies that use study designs facilitating the investigation of the mechanisms underlying the determinants. Third, more research is needed to investigate how de-implementation determinants relate to existing determinant frameworks, including CFIR. There is especially a lack of studies that address determinants related to the process domain. Fourth, the existing literature illustrates a divergent effect of determinants. More research is needed to examine how determinants differ depending on the type of LVC practice. Fifth, the majority of the identified studies were conducted in the USA and the external validity of these studies is unclear. Thus, studies on de-implementation in other countries, including low- and middle-income countries, and health care systems (e.g., with different funding structures) are warranted.

\section{Methodological considerations}

The applied methods had multiple strengths: the search was designed and performed with the university library and a structured process was applied [21]. Furthermore, screening and coding determinants were conducted by at least two authors and team discussions were applied to resolve queries. The data charting was conducted individually which implies a risk that discrepancies in data charting were missed. To mitigate this we performed a thorough testing of the data charting form and held team discussions about difficulties that occurred. Further, one of the authors performed a quality control of all the extracted data. It should be noted that although the coding and categorisation of determinants were conducted using an inductive approach the authors have considerable pre-existing knowledge concerning implementation determinant frameworks and implementation terminology which likely has influenced the understanding of the determinants as well as the labelling of categories.

The study has some limitations. The search for the review was conducted up to June 2018. This means that newer publications are not included in the results. The inconsistent terminology for LVC and de-implementation [25] and the range of clinical fields that the studies were conducted in entails a risk that studies may have been missed. Moreover, the review covers literature only in English and published in peer-reviewed sources. Also, the degree of evidence for the practice being of low value may differ between studies, as we relied on the referencing the studies made to clinical guidelines. Studies of different designs were included in the review to provide a broad overview of reported determinants for the use of LVC and de-implementation of LVC. Therefore, the review does not provide information about the strength of the associations of the identified determinants. Furthermore, the amount of information about the identified determinants in the included studies varied considerably which sometimes limited the information that could be reported in this review.

\section{Conclusion}

This scoping review provides the first compilation of determinants for the use of LVC as well as deimplementation of LVC. The identified determinants largely overlap with existing implementation frameworks, although patient expectations and professionals' fear of malpractice appear to be more prominent determinants for the use and de-implementation of LVC. Thus, existing implementation determinant frameworks may require adaptation to be transferable to de-implementation. Strategies for reducing the use of LVC should specifically consider determinants for the use of LVC and de-implementation of LVC.

\section{Supplementary Information}

The online version contains supplementary material available at https://doi. org/10.1186/s43058-021-00110-3.

Additional file 1. Preferred Reporting Items for Systematic reviews and Meta-Analyses extension for Scoping Reviews (PRISMA-SCR) Checklist

Additional file 2. Documentation of search strategies

Additional file 3. Definitions of sub-categories and overview of individual codes included in each sub-category

Additional file 4. Table A. Information about included studies on determinants for the use of LVC. Table B. Information about included studies on determinants for the de-implementation of LVC

Abbreviations

LVC: Low-value care; CFIR: Consolidated Framework for Implementation Research 


\section{Acknowledgements}

The authors would like to thank Carl Gornitzki, Sabina Gillsund and GunBrit Knutssön at Karolinska Institutet university library, who assisted with the development of the search strategy and with the data base searches.

\section{Authors' contributions}

$\mathrm{HA}, \mathrm{HH}, \mathrm{UVTS}, \mathrm{PN}$ and SI designed the study. HA, SI, IM and JD conducted the abstract screening and HA, SI, IM, JD, PN conducted the full-text screening and data charting. HA, SI, HH, UvTS and PN coded and categorised the extracted data. HA drafted the first version of the article with assistance from $\mathrm{HH}, \mathrm{SI}$ and IM. All authors discussed the draft, revised it and approved the final manuscript

\section{Funding}

This study is funded by the Swedish Research Council for Health, Working life and Welfare (FORTE) (project no. 2017-00449). Open Access funding provided by Karolinska Institute.

\section{Availability of data and materials}

The datasets used will be available from the corresponding author on reasonable request.

\section{Ethics approval and consent to participate}

Not applicable.

\section{Consent for publication}

Not applicable.

\section{Competing interests}

The authors declare that they have no competing interests.

\section{Author details}

'Procome Research Group, Medical Management Centre, Department of Learning, Informatics, Management and Ethics, Karolinska Institutet, SE 171 77 Stockholm, Sweden. ${ }^{2}$ Unit for Implementation and Evaluation, Center for Epidemiology and Community Medicine (CES), Stockholm Region, SE 17129 Stockholm, Sweden. ${ }^{3}$ Department of Health, Medical and Caring Sciences, Division of Society and Health, Linköping University, Linköping, Sweden. ${ }^{4}$ School of Health, Care and Social Welfare, Mälardalen University, Box 883, 72123 Västerås, Sweden.

\section{Received: 19 May 2020 Accepted: 14 January 2021}

\section{Published online: 04 February 2021}

\section{References}

1. Brownlee S, Chalkidou K, Doust J, Elshaug AG, Glasziou P, Heath I, et al. Evidence for overuse of medical services around the world. The Lancet. 2017:390(10090):156-68.

2. Verkerk EW, Tanke MAC, Kool RB, van Dulmen SA, Westert GP. Limit, lean or listen? A typology of low-value care that gives direction in deimplementation. International journal for quality in health care : journal of the International Society for Quality in Health Care. 2018;30(9):736-9.

3. Charlesworth CJ, Meath TH, Schwartz AL, McConnell KJ. Comparison of lowvalue care in Medicaid vs commercially insured populations. JAMA internal medicine. 2016;176(7):998-1004.

4. Mason DJ. Choosing wisely: changing clinicians, patients, or policies? JAMA. 2015;313(7):657-8

5. ABIM Foundation. The "Top 12" Recommendations That Are Reducing Overuse 2018. Available from: https://www.choosingwisely.org/resources/ updates-from-the-field/the-top-12-recommendations-that-are-reducingoveruse/. [cited 202030 December 2020].

6. Elshaug AG, McWilliams JM, Landon BE. The value of low-value lists. JAMA. 2013:309(8):775-6.

7. Schwartz AL, Landon BE, Elshaug AG, Chernew ME, McWilliams JM. Measuring low-value care in Medicare. JAMA Intern Med. 2014;174(7): 1067-76.

8. Van Bodegom-Vos L, Davidoff F, Marang-Van De Mheen PJ. Implementation and de-implementation: two sides of the same coin? BMJ Qual Saf. 2017; 26(6):495-501.

9. Levinson W, Kallewaard M, Bhatia RS, Wolfson D, Shortt S, Kerr EA. 'Choosing Wisely': a growing international campaign. BMJ Qual Saf. 2015;24(2):167-74.
10. Rosenberg A, Agiro A, Gottlieb M, Barron J, Brady P, Liu Y, et al. Early trends among seven recommendations from the Choosing Wisely campaign JAMA Intern Med. 2015;175(12):1913-20.

11. Nilsen P. Making sense of implementation theories, models and frameworks, Implement Sci. 2015;10(53).

12. Damschroder LJ, Aron DC, Keith RE, Kirsh SR, Alexander JA, Lowery JC. Fostering implementation of health services research findings into practice: a consolidated framework for advancing implementation science. Implement Sci. 2009;4(50),

13. Rycroft-Malone J. Promoting action on research implementation in health services (PARIHS). In: Jo R-M, Tracey B, editors. Models and frameworks for implementing evidence-based practice: linking evidence to action: John Wiley \& Sons; 2010.

14. Cane J, O'Connor D, Michie S. Validation of the theoretical domains framework for use in behaviour change and implementation research. Implement Sci. 2012;7(37).

15. Upvall MJ, Bourgault AM. De-implementation: a concept analysis. Nurs Forum. 2018;53(3):376-82.

16. Nilsen $\mathrm{P}$, Ingvarsson $\mathrm{S}$, Hasson $\mathrm{H}$, von Thiele Schwarz U, Augustsson H. Theories, models, and frameworks for de-implementation of low-value care: a scoping review of the literature. Implementation Research and Practice. 2020;1(Jan-Dec 2020):1-15.

17. Powell AA, Bloomfield HE, Burgess DJ, Wilt TJ, Partin MR. A conceptual framework for understanding and reducing overuse by primary care providers. Med Care Res Rev. 2013;70(5):451-72.

18. Harris C, Green S, Elshaug AG. Sustainability in Health care by Allocating Resources Effectively (SHARE) 10: operationalising disinvestment in a conceptual framework for resource allocation. BMC Health Serv Res. 2017;17(632).

19. Morgan DJ, Leppin AL, Smith CD, Korenstein D. A practical framework for understanding and reducing medical overuse: conceptualizing overuse through the patient-clinician interaction. J Hosp Med). 2017;12(5):346-51.

20. Parchman ML, Henrikson NB, Blasi PR, Buist DS, Penfold R, Austin B, et al. Taking action on overuse: cOreating the culture for change. Healthcare. 2017;5(4):199-203.

21. Arksey H, O'Malley L. Scoping studies: towards a methodological framework. Int J Soc Res Methodol. 2005:8(1):19-32.

22. Tricco AC, Lillie E, Zarin W, O'Brien KK, Colquhoun H, Levac D, et al. PRISMA extension for scoping reviews (PRISMA-SCR): checklist and explanation. Ann Intern Med. 2018;169(7):467-73.

23. Hasson $H$, Nilsen P, Augustsson $H$, von Thiele Schwarz U. Empirical and conceptual investigation of de-implementation of low-value care from professional and health care system perspectives: a study protocol. Implement Sci. 2018;13(67).

24. The Joanna Briggs Institute. The Joanna Briggs Institute Reviewers Manual 2015: Methodology for JBI scoping reviews. The Joanna Briggs Institute, 2015.

25. Niven DJ, Mrklas KJ, Holodinsky JK, Straus SE, Hemmelgarn BR, Jeffs LP, et al. Towards understanding the de-adoption of low-value clinical practices: a scoping review. BMC Med. 2015;13(255).

26. Ouzzani M, Hammady H, Fedorowicz Z, Elmagarmid A. Rayyan — a web and mobile app for systematic reviews. Syst Rev. 2016;5(210).

27. Elo $\mathrm{S}$, Kyngäs $\mathrm{H}$. The qualitative content analysis process. Journal of advanced nursing. 2008;62(1):107-15.

28. Voorn VMA, van Bodegom-Vos L, So-Osman C. Towards a systematic approach for (de)implementation of patient blood management strategies. Transfus Med. 2018;28(2):158-67.

29. Dallas A, Magin P, Morgan S, Tapley A, Henderson K, Ball J, et al. Antibiotic prescribing for respiratory infections: a cross-sectional analysis of the ReCEnT study exploring the habits of early-career doctors in primary care. Fam Pract. 2014;32(1):49-55.

30. Bhatia RS, Bouck Z, Ivers NM, Mecredy G, Singh J, Pendrith C, et al. Electrocardiograms in low-risk patients undergoing an annual health examination. JAMA Intern Med. 2017;177(9):1326-33.

31. Faustino CG, Passarelli MC, Jacob-Filho W. Potentially inappropriate medications among elderly Brazilian outpatients. Sao Paulo Med J. 2013;131(1):19-26.

32. Simos D, Hutton B, Graham ID, Arnaout A, Caudrelier JM, Clemons M. Imaging for metastatic disease in patients with newly diagnosed breast cancer: are doctor's perceptions in keeping with the guidelines? J Eval Clin Pract. 2015;21(1):67-73.

33. Ramsey SD, Henry NL, Gralow JR, Mirick DK, Barlow W, Etzioni R, et al. Tumor marker usage and medical care costs among older early-stage breast cancer survivors. J Clin Oncol. 2015;33(2):149-55. 
34. Dekker AR, Verheij TJ, van der Velden AW. Inappropriate antibiotic prescription for respiratory tract indications: most prominent in adult patients. Fam Pract. 2015;32(4):401-7.

35. Irfan N, Brooks A, Mithoowani S, Celetti SJ, Main C, Mertz D. A controlled quasi-experimental study of an educational intervention to reduce the unnecessary use of antimicrobials for asymptomatic bacteriuria. PLoS ONE. 2015;10(7):e0132071.

36. Cope AL, Francis NA, Wood F, Chestnutt IG. Antibiotic prescribing in UK general dental practice: a cross-sectional study. Community Dent Oral Epidemiol. 2016:44(2):145-53.

37. Selby K, Cornuz J, Cohidon C, Gaspoz J-M, Senn N. How do Swiss general practitioners agree with and report adhering to a top-five list of unnecessary tests and treatments? Results of a cross-sectional survey. Eur J Gen Pract. 2018;24(1):32-8.

38. Lin MP, Nguyen T, Probst MA, Richardson LD, Schuur JD. Emergency physician knowledge, attitudes, and behavior regarding ACEP's Choosing Wisely Recommendations: a survey study. Acad Emerg Med. 2017;24(6):668-75

39. Kachalia A, Berg A, Fagerlin A, Fowler KE, Hofer TP, Flanders SA, et al. Overuse of testing in preoperative evaluation and syncope: a survey of hospitalists. Ann Intern Med. 2015;162(2):100-8.

40. Sawan M, Jeon Y-H, Fois RA, Chen TF. Exploring the link between organizational climate and the use of psychotropic medicines in nursing homes: a qualitative study. Res Social Adm Pharm. 2017;13(3):513-23.

41. Kerns JW, Winter JD, Winter KM, Boyd T, Etz RS. Primary care physician perspectives about antipsychotics and other medications for symptoms of dementia. The Journal of the American Board of Family Medicine. 2018; 31(1):9-21.

42. Ryskina KL, Holmboe ES, Bernabeo E, Werner RM, Shea JA, Long JA. US internists' awareness and use of overtreatment guidelines: a national survey. Am J Manag Care. 2017;23(7):420-7.

43. Munoz-Plaza CE, Parry C, Hahn EE, Tang T, Nguyen HQ, Gould MK, et al. Integrating qualitative research methods into care improvement efforts within a learning health system: addressing antibiotic overuse. Health Res Policy Syst. 2016;14(63).

44. Redwood R, Knobloch MJ, Pellegrini DC, Ziegler MJ, Pulia M, Safdar N. Reducing unnecessary culturing: a systems approach to evaluating urine culture ordering and collection practices among nurses in two acute care settings. Antimicrob Resist Infect Control. 2018;7(4).

45. Hines JZ, Sewell $J L$, Sehgal NL, Moriates C, Horton CK, Chen AH. "Choosing wisely" in an academic department of medicine. Am J Med Qual. 2014;30(6): 566-70.

46. Sedrak MS, Patel MS, Ziemba JB, Murray D, Kim EJ, Dine CJ, et al. Residents' self-report on why they order perceived unnecessary inpatient laboratory tests. J Hosp Med. 2016:11(12):869-72.

47. Bishop TF, Cea M, Miranda Y, Kim R, Lash-Dardia M, Lee Jl, et al. Academic physicians' views on low-value services and the Choosing Wisely campaign: A qualitative study. Healthcare. 2017;5(1-2):17-22

48. Alber K, Kuehlein T, Schedlbauer A, Schaffer S. Medical overuse and quaternary prevention in primary care - a qualitative study with general practitioners. BMC Fam Pract. 2017;18(1):99.

49. Dempsey PP, Businger AC, Whaley LE, Gagne JJ, Linder JA. Primary care clinicians' perceptions about antibiotic prescribing for acute bronchitis: a qualitative study. BMC Fam Pract. 2014;15:194.

50. Han PK, Klabunde CN, Noone AM, Earle CC, Ayanian JZ, Ganz PA, et al. Physicians' beliefs about breast cancer surveillance testing are consistent with test overuse. Med Care. 2013;51(4):315-23.

51. Hong AS, Ross-Degnan D, Zhang F, Wharam JF. Clinician-level predictors for ordering low-value imaging. JAMA Intern Med. 2017;177(11):1577-85.

52. Grover M, Abraham N, Chang YH, Tilburt J. Physician cost consciousness and use of low-value clinical services. J Am Board Fam Med. 2016;29(6): 785-92.

53. Chan PS, Rao SV, Bhatt DL, Rumsfeld JS, Gurm HS, Nallamothu BK, et al. Patient and hospital characteristics associated with inappropriate percutaneous coronary interventions. J Am Coll Cardiol. 2013;62(24):2274-81.

54. Schmidt ML, Spencer MD, Davidson LE. Patient, provider, and practice characteristics associated with inappropriate antimicrobial prescribing in ambulatory practices. Infect Control Hosp Epidemiol. 2018;39(3): 307-15.

55. Kepka D, Breen N, King JB, Meissner HI. R, KB, et al. Demographic factors associated with overuse of Pap testing. Am J Prev Med. 2014;47(5):629-33.
56. Doukky R, Hayes K, Frogge N, Nazir NT, Collado FM, Williams KA Sr. Impact of insurance carrier, prior authorization, and socioeconomic status on appropriate use of SPECT myocardial perfusion imaging in private community-based office practice. Clin Cardiol. 2015;38(5):267-73.

57. Ladapo JA, Blecker S, Douglas PS. Physician decision making and trends in the use of cardiac stress testing in the United States: an analysis of repeated cross-sectional data. Ann Intern Med. 2014;161(7):482-90.

58. Barnett ML, Linder JA, Clark CR, Sommers BD. Low-value medical services in the safety-net population. JAMA Intern Med. 2017;177(6):829-37.

59. Pickles K, Carter SM, Rychetnik L, Entwistle VA. Doctors' perspectives on PSA testing illuminate established differences in prostate cancer screening rates between Australia and the UK: a qualitative study. BMJ Open. 2016;6(12): e011932.

60. Ellis SD, Nielsen ME, Carpenter WR, Jackson GL, Wheeler SB, Liu H, et al. Gonadotropin-releasing hormone agonist overuse: urologists' response to reimbursement and characteristics associated with persistent overuse. Prostate Cancer Prostatic Dis. 2015;18(2):173-81.

61. Beuscart JB, Genin M, Dupont C, Verloop D, Duhamel A, Defebvre MM, et al. Potentially inappropriate medication prescribing is associated with socioeconomic factors: a spatial analysis in the French Nord-Pas-de-Calais Region. Age Ageing. 2017:46(4):607-13.

62. Colla CH, Morden NE, Sequist TD, Schpero WL, Rosenthal MB. Choosing wisely: prevalence and correlates of low-value health care services in the United States. J Gen Intern Med. 2015;30(2):221-8.

63. Colla CH, Sequist TD, Rosentha MB, Schpero WL, Gottlieb DJ, Morden NE. Use of non-indicated cardiac testing in low-risk patients: Choosing Wisely. BMJ Qual Saf. 2015;24(2):149-53.

64. Makarov DV, Hu EY, Walter D, Braithwaite RS, Sherman S, Gold HT, et al. Appropriateness of prostate cancer imaging among veterans in a delivery system without incentives for overutilization. Health Serv Res. 2015;51(3): 1021-51.

65. Silverman M, Povitz M, Sontrop JM, Li L, Richard L, Cejic S, et al. Antibiotic prescribing for nonbacterial acute upper respiratory infections in elderly persons. Ann Intern Med. 2017;166(11):765-74.

66. Nuytten A, Behal H, Duhamel A, Jarreau PH, Mazela J, Milligan D, et al. Evidence-based neonatal unit practices and determinants of Postnatal corticosteroid-use in preterm births below 30 weeks ga in Europe. A population-based cohort study. PLoS ONE. 2017;12(1).

67. Cuba Fuentes MS, Zegarra Zamalloa CO, Reichert S, Gill D. Attitudes, perceptions and awareness concerning quaternary prevention among family doctors working in the Social Security System, Peru: a cross-sectional descriptive study. Medwave. 2016;16(3):e6433.

68. Chalmers JD, Tebboth A, Gayle A, Ternouth A, Ramscar N. Determinants of initial inhaled corticosteroid use in patients with GOLD A/B COPD: a retrospective study of UK general practice. NPJ Prim Care Respir Med. 2017; 27(1):1-8

69. Makarov DV, Soulos PR, Gold HT, Yu JB, Sen S, Ross JS, et al. Regional-Level Correlations in Inappropriate Imaging Rates for Prostate and Breast Cancers: Potential Implications for the Choosing Wisely Campaign. JAMA Oncol. 2015;1(2):185-94.

70. Mafi JN, Wee CC, Davis RB. L, on BE. Association of primary care practice location and ownership with the provision of low-value care in the United States. JAMA Intern Med. 2017;177(6):838-45.

71. Wang DE, Lam DJ, Bellmunt AM, Rosenfeld RM, Ikeda AK, Shin JJ. Intranasal steroid use for otitis media with effusion: ongoing opportunities for quality improvement. Otolaryngol Head Neck Surg. 2017;157(2):289-96.

72. Barlam TF, Morgan JR, Wetzler LM, Christiansen CL, Drainoni ML. Antibiotics for respiratory tract infections: a comparison of prescribing in an outpatient setting. Infect Control Hosp Epidemiol. 2015;36(2):153-9.

73. Singh A, Bodukam V, Saigal K, Bahl J, Wang Y, Hanlon A, et al. Identifying risk factors associated with inappropriate use of acid suppressive therapy at a community hospital. Gastroenterol Res Pract. 2016;2016(Article id:1973086).

74. Jiron M, Pate V, Hanson LC, Lund JL, Jonsson Funk M, Sturmer T. Trends in prevalence and determinants of potentially inappropriate prescribing in the United States: 2007 to 2012. J Am Geriatr Soc. 2016;64(4):788-97.

75. Kondo N, Nakamura F, Yamazaki S, Yamamoto Y, Akizawa T, Akiba T, et al. Prescription of potentially inappropriate medications to elderly hemodialysis patients: prevalence and predictors. Nephrol Dial Transplant. 2014;30(3):498-505.

76. Rosenthal MB, Colla CH, Morden NE, Sequist TD, Mainor AJ, et al. Overuse and insurance plan type in a privately insured population. Am J Manag Care. 2018;24(3):140-6. 
77. Bell HT, Steinsbekk A, Granas AG. Factors influencing prescribing of fall-riskincreasing drugs to the elderly: A qualitative study. Scand J Prim Health Care. 2013;33(2):107-14.

78. Amos TB, Keith SW, Del Canale S, Orsi P, Maggio M, Baccarini S, et al. Inappropriate prescribing in a large community-dwelling older population: a focus on prevalence and how it relates to patient and physician characteristics. J Clin Pharm Ther. 2015;40(1):7-13.

79. Lipitz-Snyderman A, Sima CS, Atoria CL, Elkin EB, Anderson C, Blinder V, et al. Physician-driven variation in nonrecommended services among olde adults diagnosed with cancer. JAMA Intern Med. 2016;176(10):1541-8.

80. Kruse GR, Khan SM, Zaslavsky AM, Ayanian JZ, Sequist TD. Overuse of colonoscopy for colorectal cancer screening and surveillance. J Gen Intern Med. 2015;30(3):277-83.

81. Clyne B, Cooper JA, Hughes CM, Fahey T, Smith SM, Team O-Ss. 'Potentially inappropriate or specifically appropriate?' Qualitative evaluation of general practitioners views on prescribing, polypharmacy and potentially inappropriate prescribing in older people. BMC Fam Pract. 2016;17(1):109.

82. Sawan MJ, Jeon YH, Fois RJ, Chen TF. A qualitative study exploring visible components of organizational culture: what influences the use of psychotropic medicines in nursing homes? Int Psychogeriatr. 2016;28(10): 1725-35.

83. Means AR, Weaver MR, Burnett SM, Mbonye MK, Naikoba S, RS MC, et al. Correlates of inappropriate prescribing of antibiotics to patients with malaria in Uganda. PLoS ONE. 2014;9(2):e90179.

84. Specchia ML, La Torre G, Calabro GE, Villari P, Grilli R, Federici A, et al. Disinvestment in cancer care: a survey investigating European countries' opinions and views. Eur J Public Health. 2018;28(6):987-2

85. Zikmund-Fisher BJ, Kullgren JT, Fagerlin A, Klamerus ML, Bernstein SJ, Kerr EA. Perceived barriers to implementing individual Choosing Wisely ${ }^{\oplus}$ recommendations in two national surveys of primary care providers. J Gen Intern Med. 2017;32(2):210-7.

86. Barnes GD, Misirliyan S, Kaatz S, Jackson EA, Haymart B, Kline-Rogers E, et al. Barriers and facilitators to reducing frequent laboratory testing for patients who are stable on warfarin: a mixed methods study of de-implementation in five anticoagulation clinics. Implement Sci. 2017;12(87).

87. Greene SE, Massone R. A survey of emergency medicine residents perspectives of the choosing wisely campaign. Am J Emerg Med. 2015; 33(6):853-5.

88. Buist DS, Chang E, Handley M, Pardee R, Gundersen G, Cheadle A, et al. Primary care clinicians' perspectives on reducing low-value care in an integrated delivery system. Perm J. 2016;20(1):41-6.

89. Silverstein W, Lass E, Born K, Morinville A, Levinson W, Tannenbaum C. A survey of primary care patients' readiness to engage in the deadoption practices recommended by Choosing Wisely Canada. BMC Res Notes. 2016;9(301).

90. Voorn VM, Marang-van de Mheen PJ, Wentink MM, Kaptein AA, Koopmanvan Gemert AW, So-Osman C, et al. Perceived barriers among physicians for stopping non-cost-effective blood-saving measures in total hip and total knee arthroplasties. Transfusion. 2014;54(10 pt2):2598-607.

91. Robert G, Harlock J, Williams I. Disentangling rhetoric and reality: an international Delphi study of factors and processes that facilitate the successful implementation of decisions to decommission healthcare services. Implement Sci. 2014:9(123)

92. Greenwood BN, Agarwal R, Agarwal R, Gopal A. The when and why of abandonment: the role of organizational differences in medical technology life cycles. Manage Sci. 2016;63(9):2948-66.

93. Davidson KW, Ye S, Mensah GA. Commentary: De-implementation science: a virtuous cycle of ceasing and desisting low-value care before implementing new high value care. Ethn Dis. 2017;27(4):463-8.

94. Birken SA, Powell BJ, Shea CM, Haines ER, Kirk MA, Leeman J, et al. Criteria for selecting implementation science theories and frameworks: results from an international survey. Implement Sci. 2017;12(124).

95. Durlak JA, DuPre EP. Implementation matters: a review of research on the influence of implementation on program outcomes and the factors affecting implementation. Am J Community Psychol. 2008;41:327-50.

96. Greenhalgh T, Robert G, Macfarlane F, Bate P, Kyriakidou O. Diffusion of innovations in service organizations: systematic review and recommendations. Milbank Q. 2004;82(4):581-629.

97. Organization. WH. Global action plan on antimicrobial resistance. 2015

98. Rockwell M, Kraak V, Hulver M, Epling J. Clinical management of low vitamin D: a scoping review of physicians' practices. Nutrients. 2018;10(493).
99. Choosing Wisely. American Society for Clinical Pathology: don't perform population based screening for 25-OH-vitamin D deficiency. http://www. choosingwisely.org/clinician-lists/american-society-clinical-pathologypopulation-based-screening-for-vitamin-d-deficiency/2013 [cited 2020 March 31].

100. Norton WE, Chambers DA. Unpacking the complexities of de-implementing inappropriate health interventions. Implement Sci. 2020;15(2).

101. Goss S, Rowland N. Getting evidence into practice. In: Goss NRS, editor. Evidence Based Counselling and Psychological Therapies London: Routledge; 2000. p. 191-205.

102. Buchen $I H$. An organizational new year's resolution: to unlearn. National Productivity Review. 1998;18(1):1-4.

103. Gupta DM, Boland RJ, Aron DC. The physician's experience of changing clinical practice: a struggle to unlearn. Implement Sci. 2017;12(28).

104. Helfrich CD, Rose AJ, Hartmann CW, van Bodegom-Vos L, Graham ID, Wood SJ, et al. How the dual process model of human cognition can inform efforts to de-implement ineffective and harmful clinical practices: a preliminary model of unlearning and substitution. J Eval Clin Pract. 2018; 24(1):198-205.

105. Schein EH. Organizational Culture and Leadership. 3rd Edition. ed. San Francisco: Jossey-Bass; 2004.

106. CFIRguide. Process. https://cfirguide.org/constructs/process/. Accessed 23 June 2020.

\section{Publisher's Note}

Springer Nature remains neutral with regard to jurisdictional claims in published maps and institutional affiliations.
Ready to submit your research? Choose BMC and benefit from:

- fast, convenient online submission

- thorough peer review by experienced researchers in your field

- rapid publication on acceptance

- support for research data, including large and complex data types

- gold Open Access which fosters wider collaboration and increased citations

- maximum visibility for your research: over $100 \mathrm{M}$ website views per year

At BMC, research is always in progress.

Learn more biomedcentral.com/submissions 